\title{
Aerosol antibiotic resistance gene dissemination among hospital departments by culture-facilitated comparative metagenomics
}

\section{Xiang Li}

Southern University of Science and Technology

\section{Ziqi Wu}

Southern University of Science and Technology

\section{Chenyuan Dang}

Southern University of Science and Technology

\section{Miao Zhang}

Southern University of Science and Technology

\section{Bixi Zhao}

Southern University of Science and Technology

\section{Zhanwen Cheng}

Southern University of Science and Technology

\section{Liming Chen}

Southern University of Science and Technology

\section{Zhenfeng Zhong}

Peking University Shenzhen Hospital

\section{Yuhui Ye}

Peking University Shenzhen Hospital

YuXia ( $\nabla$ xiay@sustech.edu.cn)

The University of Manchester Faculty of Biology Medicine and Health https://orcid.org/0000-00032842-6639

\section{Research}

Keywords: built-in hospital environment, aerosol, ARGs, culture-facilitated comparative metagenomics

Posted Date: January 8th, 2020

DOl: https://doi.org/10.21203/rs.2.20350/v1

License: (c) (1) This work is licensed under a Creative Commons Attribution 4.0 International License. 



\section{Aerosol antibiotic resistance gene dissemination}

\section{2 among hospital departments by culture-facilitated}

3 comparative metagenomics

4

5 Xiang $\mathrm{Li}^{\mathrm{a}, \mathrm{b}}$, Ziqi Wu ${ }^{\mathrm{b}}$, Chenyuan Dang ${ }^{\mathrm{b}}$, Miao Zhang ${ }^{\mathrm{b}}$, Bixi Zhao ${ }^{\mathrm{b}}$, Zhanwen

6 Cheng ${ }^{\mathrm{b}}$, Liming Chen ${ }^{\mathrm{b}}$, Zhenfeng Zhong ${ }^{\mathrm{c}}$, Yuhui $\mathrm{Ye}^{\mathrm{c}}$, Yu Xia ${ }^{\mathrm{a}, \mathrm{b}^{*}}$

8 Email addresses:

9 Xiang Li: lix36@sustech.edu.cn; Ziqi Wu: ziqi.wu@bio.ku.dk; Chenyuan Dang: 10 dangcy@sustech.edu.cn; Miao Zhang: 11849070@mail.sustech.edu.cn; Bixi 11 Zhao: 11930297@mail.sustech.edu.cn; Zhanwen Cheng:

12 11930869@mail.sustech.edu.cn; Liming Chen: chenlm@pku.edu.cn;

13 Zhenfeng Zhong: 33283374@qq.com; Yuhui Ye: 784748536@qq.com

14 Address all correspondence and requests to:

15 "Yu Xia, PhD, School of Environmental Science and Engineering, Southern 16 University of Science and Technology, Shenzhen, Guangdong 518055, China.

17 Email: xiay@sustech.edu.cn.

18 Institutional addresses:

$19{ }^{a}$ Guangdong Provincial Key Laboratory of Soil and Groundwater Pollution 20 Control, School of Environmental Science and Engineering, Southern 21 University of Science and Technology, Shenzhen, 518055, China

$22{ }^{\mathrm{b}}$ International Center for Antibiotic Resistance in the Environment, School of 23 Environmental Science and Engineering, Southern University of Science and 24 Technology, Shenzhen, 518055, China

$25{ }^{\mathrm{c}}$ Shenzhen Hospital of Peking University, 1120 Lianhua Rd., Futian, Shenzhen, 26 Guangdong, China, 518036 


\section{Abstract}

28 Background: Genes make microorganisms resistant to antibiotics are

29 overwhelmingly present in environments, and those in the air seem to

30 consistently worsen human health, especially in built-in hospitals. In this study,

31 we sampled two distinct seasonal solid-, liquid- and gaseous-state samples in

32 a large occupied hospital, and mainly aim to study the temporal and spatial

33 micro-ecology and antibiotic resistance gene (ARG) distribution patterns within

34 hospital settings using culture-facilitated comparative metagenomics in

35 combinations with other widely applied methods.

36 Results: Hospital aerosol resistome showed concentration of 0.00042 copies

37 per $16 \mathrm{~S}$ rRNA gene which was comparable to that of drinking water $(0.00024$ copies/16S rRNA). Winter aerosols showed higher resistome concentration and exhibited similar distribution patterns among different departments; while

40 evident microbial accumulation and stronger level of inter-species ARG

41 exchange was observed in summer aerosol samples. Network analysis and

42 cultural isolate whole genome sequencing results confirmed some of these

43 exchanges were mediated via plasmids. Additionally, ARGs and microbial

44 community source tracking results illustrated Outpatient hall could serve as a

45 major resistome pollution source all year long.

46 Conclusions: The state-of-art metagenomics analyses and culture facilitated 
47 sequencing with strict cutoff guarantee the result accuracy of resistome pattern

48 observed in built-in hospital environment, and our novel genotype sharing

49 network confirmed the plasmid-mediated transferring mechanism in hospital

50 air for the first time. Overall, this study illustrated valuable and quality hospital

51 aerosol dissemination patterns and our effort will encourage more

52 metagenomics applications in hospital studies.

53 Keywords: built-in hospital environment, aerosol, ARGs, culture-facilitated

54 comparative metagenomics

\section{Background}

56 Antibiotics were invented to preventing infections and saving lives [1]. However,

57 with the defensive mechanisms evolved from microorganisms, the genes

58 make microorganisms resistant are "effectively weakening" and

59 overwhelmingly present in environments after extensive applications

60 worldwide [2,3]. Not only waterborne and soil related resistant microbes are

61 threatening our health, but aerosol ARG pollution is a serious concern. Some

62 research groups are exploring ARGs from the air we breathe, and those

63 resistances seem to closely relate to air quality parameters. For instance, Li et

64 al. [4] showed $\mathrm{PM}_{2.5}$ possessed high concentration of $A R G$ against $\beta$-lactam,

65 quinolone, macrolide, tetracycline, sulfonamide, aminoglycoside and 
66 vancomycin in China. Xie et al. [5] also noticed seasonal ARG variations

67 across industrial-urban-rural areas on an annual basis.

68 Humans are tethered to the built-in environment very closely. From the hospital

69 we are born in to the homes and offices we live and work in, the built-in

70 environment has become the most intimate ecosystem for humans. Lax et al.

71 [6-8] performed a wealth of work and provided valuable datasets related to the

72 indoor micro-ecology. They revealed human microbiota can influence indoor

73 microbial communities and also be shaped by built-in environment. Notably,

74 micro-ecology associated with hospital settings arguably has the most

75 profound implications because hospital-acquired infections (HAls) have always

76 been considered as one of the main causes of patient deaths $[7,9,10]$.

77 Therefore, lots of effort weighted on determining how microorganisms colonize,

78 persist, and change in the hospital indoor environment. Many previous studies

79 have identified dominant hospital-associated pathogens (HAPs) and

80 summarized their putative routes of transmissions. Those sources mainly

81 include physicians' and staff's clothing [11-13], stethoscopes [14], phones [15-

82 18], keyboards [19] and even patient bedrails [8]. In any case, the solid

83 surfaces accumulate a large spectrum of microbiota, which naturally favors

84 horizontal gene transfer (HGT) via mobile genetic elements (MGEs). Patients

85 can acquire ARGs by "direct contact", but those "invisible aerosols" also have 
86 the chances entering into patients. To our best knowledge, the detailed

87 temporal aerosol ARG profiling variations with genus and/or species level

88 information in hospital environment seem to be lacking currently.

89 Traditional studies in researching ARGs, HAls and HAPs have relied heavily 90 on cultural isolates and molecular gene identifications [20-25]. However, the

91 'needle in a haystack' cultural, PCR and quantitative PCR (qPCR) screening

92 methods demand intensive labor and are not cost friendly. Even though high

93 magnitude qPCR technology and microarray can somehow relieve the low

94 throughput issue, the lack of assay controls and arbitrary detection threshold

95 setting are relatively invalid to resolve inaccurate and false positive signals

$96[26,27]$. Metagenomics sequencing focuses more on the qualitative patterns

97 and the signals are also comparable given same sample handling and

98 operational protocols. Also, metagenomics sequencing can generate sufficient

99 information regarding entire community and gene functionality, helping us

100 understand the potential pollution sources and distribution patterns thoroughly.

101 In this study, we mainly surveyed one-year aerosol samples from three

102 different departments in a large-scale municipal hospital. Besides aerosols, we

103 also collected water and hospital surface samples. We applied cultivation,

104 PCR, qPCR, Nanopore sequencing and Illumina metagenomics approaches to:

105 1) identify and compare ARGs and associated carriers in different departments; 
2) study the temporal and spatial microbial community and functionality

107 variations; 3) emphasize aerosol ARG distribution patterns within hospital.

\section{Results and discussion}

109 The prevalence of antimicrobial organisms and evaluations of applied

110 methodologies

111 We have cultivated total of 102 (12 are blank controls) antibiotic selective LB

112 plates targeting 15 samples (aerosol, water and surface samples) from 6

113 departments. All negative controls (no antibiotic added) showed colony and/or

114 plaque morphology. From Table S1, we can see tetracycline and erythromycin

115 had highest detection rate in all samples (both 40\%), and other antibiotic

116 detections were not far behind. The antimicrobial organisms were all bacterial

117 colonies in water samples of the Outpatient hall (M_WO) and door handles

118 seemed to mostly have tetracycline resistance in eukaryotic cells. Notably, the

119 winter and summer aerosol samples in Pediatrics department $\left(P \_A\right)$ showed

120 completely different ARG carriers (from bacteria to eukaryotes). Antibiotic

121 resistances were commonly detected using cultural method and some of the

122 samples have resistances from both bacterial and eukaryotic cells (e.g., O_A1

123 and P_A2). Equal number of bacterial colonies and eukaryotic plaques (20\%)

124 were found from all samples (blank control excluded). We also amplified total 
125 of 21 samples, including aerosol, swabs and water samples by full length rRNA 126 PCR. 11 out of $21(52.4 \%)$ were bacterial positives, and eukaryotes showed a 127 higher detection rate $(71.4 \%)$. Culture negatives of M_A1 and M_A2 showed 128 signals using PCR. Besides $M \_A 1$ and $M \_A 2$, the swabs of $P \_D$ and $C A N \_D$ 129 samples also showed similar results, but most of the samples demonstrated 130 solid consistency with cultural isolations. In this study, cultivation and PCR 131 methods identified both bacterial and eukaryotic resistances from aerosol, with 132 comparable detection rates to water and surface samples, suggesting aerosol 133 might be a potential transition route in hospital setting, which was also 134 consistent with others $[8,20,21,50-54]$. The same $20 \%$ detections for both 135 bacterial and eukaryotic resistances suggest resistant eukaryotes might be an 136 overlook. PCR techniques showed higher detection rates (at least 12\% higher), 137 indicating PCR has better methodology sensitivity but apparently, PCR is not 138 able to distinguish live or dead cells.

139 For qPCR assays, we amplified 15 samples (Table S2), but only obtained 140 positive results from int/1, tetW and $16 \mathrm{~S}$ assays. One of the explanations might

141 be we have only applied the correct candidate primers regarding those two 142 assays since both int/1 and tetW primers used here were widely applied in 143 hospital settings (other primers were designed for non-hospital environment). 144 Therefore, our results indicated the selection of specific hospital ARG primers 
145 was necessary since ARG genotypes varied greatly [29]. All standard curves 146 (int/1, tetW and 16S) have higher $\mathrm{R}^{2}$ values $(>0.983)$, but the amplification 147 efficiencies varied from 1.1 to 2.0 . The lowest limit of detection (LOD) value is $14810^{4}$ gene copies/reaction. 13 out of 15 samples were int/1 positives (two water 149 samples not amplified), and aerosol samples showed higher concentrations 150 compared to other types (data not shown). Similar detection trends were found 151 with tetW, but we noticed two water samples were positive this time $(100 \%$ 152 detection). ARG qPCR results showed winter samples possessed relatively 153 higher concentration regarding all sample types, and aerosol samples can be 154 quantified better than water samples. One of the possible reasons could be the 155 aerosol microbes were accumulated longer on solid surfaces than water 156 samples. However, tetW was able to be identified even in water samples, 157 suggesting tetracycline was very prevalence and dominant in this hospital. We 158 also applied metagenomics approach to profiling ARGs, and the main present 159 ARG subtypes were multidrug (all year long) and clear temporal aerosol 160 distribution patterns within different departments were noticed [55]. In reality, it 161 is impossible to cultivate all antibiotic samples, but cultivation is the best way to 162 illustrate the correct antibiotic phenotypes. In our study, metagenomics 163 sequencing only generated short reads and required computational resources 164 to reconstruct the "broken" genomic fragments. Therefore, inconsistencies 
between cultural and metagenomics sequencing are unavoidable, which is supported by a newly published work [56]. The varied sensitivity and specificity

167 values from cultural, PCR and sequencing methods suggest using only one of 168 the techniques may cause inconsistent interpretations. Therefore, we also 169 applied Nanopore sequencing to confirming the ARGs that were carried by 170 either bacterial genomes or plasmids. Using Nanopore sequencing, we 171 successfully assembled 5 bacterial genomes and detected 25 potential 172 plasmid-like fragments, confirming the prevalence of ARGs and necessity of 173 applying multiple methodologies. We applied very strict rules searching those 174 plasmid-like fragments in order to generate confident interpretations. We also 175 aimed to support that sample handling and DNA extraction were able to play 176 important roles in interpreting results. For instance, some environmental 177 research groups recommended limited freeze-thaw cycles and relatively mild 178 cell lysis steps [57]. In this study, tetW qPCR had good correlation with 179 metagenomic sequencing, indicating metagenomics sequencing has the ability 180 to accurately detect gene concentrations quantitatively. However, since 181 tetracycline resistance was such dominant and concentrated in this hospital, it 182 seems sequencing may have quantitative ability only if DNA quantity and 183 quality were guaranteed. Notably, we did not identify the tetW carrier, and the 184 one of the possible reasons could be carrier fragments were "broken apart" 
during sample handling. Overall, using all widely applied methods together, we

186 proved ARGs were prevalent in almost every possible media (air, water and 187 surfaces) within hospital setting, and the winter aerosols had the most diverse 188 and concentrated ARGs in all tested departments.

\section{Aerosolized antibiotic resistance threats in this hospital}

190 From metagenomics analyses, we saw winter aerosol samples had much 191 higher ARG diversity and abundance than summer samples overall. For 192 instance, among 1208 total ARG subtypes in database, P1 had 167 hits, 193 followed by 144 detections in M1 and only 42 in O1, but P2 had 384 hits 194 followed by O2 and M2 with 357 and 344, respectively. 727 out of 1208 195 subtypes were not present in all aerosol samples, and only 36 detections were 196 found in all samples, suggesting a highly diversified aerosol resistome in 197 hospital setting. The common identified included 1 bacitracin, 2 198 aminoglycoside, 2 chloramphenicol, 2 fosmidomycin, 1 MLS, 1 sulfonamide, 1 199 unclassified and 26 multidrug subtypes. If grouped by sampling seasons, 86 200 occurred in winter (besides mentioned subtypes above, quinolone, 201 tetracenomycin, tetracycline, trimethoprim and vancomycin subtypes were 202 newly found) and only 11 in summer (only $\beta$-lactam and multidrug subtypes).

203 Notably, qPCR results showed a similar trend, and a $R^{2}$ value of 0.72 was 204 obtained between normalized qPCR (tetW per 16S rRNA gene copy) and tetW 
abundances determined by ARG-OAP (tetW per 16S rRNA gene copy). Even

206 though ANOSIM showed no significant differences among all aerosols $(p=$

207 0.67), the ordination from PCoA analysis was still able to visually separate

208 seasonal variations. In general, each department has relative unique

209 resistome especially in summer. As shown in Figure 1A, winter aerosol

210 samples clustered closely in regard to both aerosol ARG genotypes and

211 community (OTU-based), while summer samples from different departments

212 showed evident scattering. Additionally, hospital aerosol resistome is distinct

213 from other environments but closely relates to human-related samples (Figure

214 1B), highlighting the direct impact of patients visiting on hospital aerosol

215 resistome. From Table 1, the commonly present ARG carriers identified in this

216 study included genera of Escherichia, Bacteroides, Staphylococcus,

217 Corynebacterium, Micrococcus and Acinetobacter, which were also clearly

218 noticed in other studies $[20,54,55]$. Corresponding to its high patients visiting

219 and personnel exchange frequency, Outpatient hall (main entrance of the

220 hospital) holds the most diverse and concentrated carriers, emphasizing the

221 necessarity to reinforce resistome management under high visiting frequency

222 [51]. Pediatrics and Ophthalmology showed more or less similar community

223 composition (Figure $1 \mathrm{~A}$ and Figure S2) than Outpatient hall, which was also

224 confirmed by ARG source tracking results (Figure 2). Their differences with 
225 Outpatient hall especially in summer suggest the applied treatments in 226 different departments could also change aerosol bacterial communities (also 227 confirmed by functionality profiling) and resistome.

228 Top 50 MetaPhIAn2 microbial community results (species level, totaling 1235 229 species) can be seen in Figure S2. Most of the species on list are bacteria, but 230 we also detected polyomavirus and porcine type- $C$ oncovirus at relatively 231 higher abundances. In the list, genera such as Staphylococcus, Streptococcus, 232 Acinetobacter and Pseudomonas were also commonly present in hospitals. 233 For instance, some of the species were from known sources, such as mouth 234 (Streptococcus sanguinis and Rothia dentocariosa) [58,59], skin 235 (Acinetobacter Iwoffii and Propionibacterium acnes) [60,61], clinical/hospital 236 setting (Acinetobacter johnsonii, Pseudomonas stutzeri and Acinetobacter junii) $237[60,62]$ and air dust (Aerococcus viridans, Enhydrobacter aerosaccus and 238 Micrococcus luteus) [63]. From HUMAnN2 results, a clear trend of aerobic 239 lifestyle was observed in aerosol community of the main entrance at both 240 summer and winter with aerobic respiration, TCA cycle and biosynthesis (GDP 241 mannose, L-valine, fatty acid etc.) were the dominant metabolism pathways. It 242 is interesting to observe some prevalent anaerobic pathways in the aerosol of 243 summer Pediatrics department such as Sulfate reduction, homolactic 244 fermentation and pyrimidine deoxyribonucleotide, implying a non-negligible 
245 microbial accumulation in nutrient-deficit air dust in summer hospital setting.

246 But this anaerobic metabolic trend disappeared in wintertime, and this

247 probably was caused by temperature decrease. Such growing effect could

248 also be observed by a diversity analysis (see $\mathrm{SI}$ ), which the summer samples

249 tended to have simplified community with sharply reduced Shannon index than

250 winter samples (Table S3). It seems the HAls and HAPs are closely associated

251 with visitor numbers [8,51], nonetheless, we are facing severe aerosol

252 antibiotic resistance threats, especially in winter. Our results demonstrated we

253 might need to apply different strategies to address ARG contamination

254 spatially and temporally within hospital setting, especially more effort should

255 be made towards Outpatient hall.

256 Cultivation method was able to screen antibiotic resistances; PCR and qPCR

257 could provide ARG and categorical microorganism information; sequencing

258 analysis helped us with a variety of community composition and functionality

259 knowledge. However, we still need to bridge which specific microorganisms to

260 carry which specific ARGs. In our study, we had very strict rules in determining

261 potential carrier by metagenomics approach. For instance, in our novel

262 genotype sharing network analysis, we incorporated MetaPhIAn2 to re-ensure

263 the correct carriers, and we have noticed strong consistency between two

264 state-of-art sequencing analysis methods (Figure 3, Figure S2 \& S4), 
265 illustrating quality and valuable datasets were obtained in this study. Even we

266 feel confident about bacterial carriers; we are still missing eukaryotic antibiotic

267 resistance carriers. One of the possible explanations could be eukaryotic cells

268 were not studied thoroughly in current stage. The potential carriers identified

269 by rRNA gene based analysis were comparable with other work [50,64], but as

270 shown in a diversity results we only obtained class level confidence (Table S3).

271 Therefore, high resolution of eukaryotic composition databases were needed,

272 as West et al. [65] have already stepped up and more eukaryotic

273 metagenomics sequencing related effort should be made in future.

\section{ARG temporal and spatial distribution patterns}

275 In this study, we performed three types of source tracking analyses based on

276 OTU, ARG and pathogen abundances, respectively (SI File 1 \& 2). As

277 introduced, we treated two season M aerosol samples as "source" and other

278 departments as "sink" for SourceTracker. "Unknown" is a default output,

279 representing potential sources besides initial targets. From community source

280 tracking perspective (Figure 2 OTU), although Pediatrics winter aerosol (P2)

281 still detained summer sources, the overall impact of Outpatient hall source

282 increased greatly from summer (around 20\%) to winter (around 50\%); similar

283 trend was also observed in source tracking based on aerosol resistome

284 (Figure 2 ARG). M source explained more than $80 \%$ winter resistome from 
285 Pediatrics and Ophthalmology departments while it was roughly $45 \%$ in

286 summer. Such huge impact on overall aerosol community and resistome from

287 Outpatient hall/Main entrance of hospital at winter indicated a relatively

288 "transient" feature of aerosol microbiome, which was largely introduced by

289 patients visiting via the Outpatient hall $[66,67]$. This observation was in

290 accordance with the closely clustered winter resistome revealed by PCoA

291 analysis (Figure 1A). To the contrary, the summer aerosol community is more

292 "resistant" to the influence from the entrance source which probably owned to

293 the microbial accumulation revealed by previous community functional

294 analysis and in accordance with the scattered resistome by PCoA results

295 (Figure 1A). Also, in a review paper from Lax et al. [7], the authors claimed

296 opening the windows in hospital indoor rooms has been found to significantly

297 link to the reduction of the percentage of pathogenic airborne bacteria. In this

298 study, the air conditioning systems at Shenzhen city operate over half of the

299 year at summer, indicating the closed window condition happens most of the

300 summer. Therefore, we have noticed summer pathogen persistence in

301 Ophthalmology department, which is also observed by Gao et al [55].

302 Additionally, a distinct pattern was noticed from pathogen source tracking that

303 unknown sources were dominant except for E2 which showed strong evidence

304 that the summer pathogen still persistent from summer to winter underpinning 
305 the notion that persistent pathogens could dominant in hospital setting from 306 days to months [68]. Those "unknown" could be sourced from "direct contact" 307 happened within hospital as prior studies suggested [8,64]. Additionally, some 308 new opportunistic pathogens may also challenge the SourceTracker algorithm 309 because we only focused on those well-known targets at the moment. In 310 summary, ARG and community source tracking results illustrated Outpatient 311 hall could serve as a major pollution source all year long. Besides source 312 tracking, we also applied Procrustes analysis to illustrate temporal and 313 departmental variations when compared community to resistome. Even though 314 significant association still exists $(p<0.01)$ between aerosol community and 315 resistome, Procrustes analysis suggested the summer samples showed a 316 much weakened association than winter samples as indicated by a larger

317 measure of fitness by $\mathrm{M}^{2}$ value (the sum of square distance between matched

318 sample pairs in Procrustes transformation). The findings implied a higher level 319 of inter-community ARGs exchange in summer hospital aerosol. Such 320 increased ARG-HGT level probably caused by microbial accumulation in 321 summer, which aerosol did not associate with increased resistome as the 322 winter aerosol showed much higher resistome abundance and diversity than 323 summer samples.

324 To illustrate the ARG genotype exchange pattern, we constructed ARG 
genotype sharing network with over $99 \%$ similarity to a known genotype in SARG database to map the interconnection among departments based on

327 metagenome-assemblies (Figure 3 and Figure S4). Outpatient hall had largest 328 number of ARG genotypes and the network was the most complicated. Our

329 strict algorithms detected commonly present genera such as Staphylococcus, 330 Micrococcus, Streptococcus and Enterococcus in hospital setting.

331 Staphylococcus carried the largest number of ARGs (14 ARG genotypes) 332 compared to other carriers. Fewer genera were identified in Ophthalmology 333 and Pediatrics department (Figure 3), which corresponded to the results in

334 Table 1. ARGs resistant against Aminoglycoside, bacitracin, $\beta$-lactam, 335 chloramphenicol, MLS, multidrug, tetracycline, bleomycin and sulfonamide 336 were commonly detected in this study (Table 1 and Figure S4). Consistent with 337 other main techniques used in this study, winter aerosols had higher 338 concentrations overall. High abundance of Micrococcus positively correlated 339 with high ARG genotype concentrations (Table 1). Notably, most of the ARG 340 genotypes were found from both summer and winter samples. In contrast to 341 Tet40, erm(36) and InuB which were only carried by single microorganism, 342 some instances of Inter-species HGT could be confirmed in hospital aerosol 343 samples, such as, tetK HGT between Staphylococcus, which was further 344 confirmed by whole genome Nanopore sequencing (Figure S5). The most 
345 commonly shared ARG genotypes among different departments in hospital

346 aerosol mainly included aadD, CE, tetK, tet $A$, tet $Z$ and norA. Since plasmid

347 mediated CE transmission was observed between Staphylococcus and

348 Corynebacterrium within Outpatient hall in this study, we infer plasmids carried

349 CE and tetracycline resistance could possibly lead to ARG transmission

350 among departments. Meanwhile, we have successfully assembled 5 bacterial

351 genomes with Enterococcus faecalis and Staphylococcus saprophyticus being

352 assembled into complete circular format. Also, the antibiotic phenotypes all

353 corresponded to the genotypes that were identified from either genomes or

354 plasmids. As shown in Table S6, tetracycline, aminoglycoside, macrolide and

$355 \quad \beta$-lactam were all detected from these live cells, and tetracycline resistance

356 genes were frequently found. Most of the inter-species and inter-departmental

357 ARG sharing was mediated by plasmids that were previously identified to

358 associate with Staphylococcus saprophyticus, suggesting the movement of

359 Staphylococcus saprophyticus plasmid among departments may be an

360 important HAI transfer pattern and causing infections in this hospital. Genera

361 such as Staphylococcus, Xanthomonas and Enterococcus were also

362 endangering hospital environment (confirmed by both Illumina and Nanopore

363 sequencing). Nanopore sequencing results also supported tet(K) was shared

364 among hospital departments via the presence of Enterococcus and 
365 Staphylococcus. These results suggested despite the high-throughput 366 coverage offered by metagnomic sequencing, we still need to apply culture 367 isolate sequencing to confirm the existence of plasmids. Using much longer 368 read length Nanopore sequencing, we have found similar plasmid transferring 369 phenomenon among departments and assured the existence of plasmids 370 (Figure S5 \& S6). In combination of two sequencing technologies, our 371 genotype sharing network confirmed the plasmid-mediated transferring 372 mechanism in hospital air for the first time. Wealth prior studies mostly either

373 applied amplicon sequencing technologies to identify aerosolized microbial 374 communities $[8,54,64]$; or used PCR based methods to profile resistome $375[51,69]$. Also, there are some studies applied metagenomics approaches $376[50,70]$ on aerosolized microbial community in hospital, but they did not

377 connect ARGs with specific carriers or utilized correlation network analysis to 378 connect ARG carriers to bacteria [55,71]. Based on these frontier works, in 379 this study we integrated state-of-art metagenomics to localize ARGs carried by 380 specific bacteria using strict algorithm and further confirmed the ARG 381 transferring mechanism using culture-based whole genome Nanopore 382 sequencing. The microbial community and gene functionality results from 383 aerosol metagenomics results identified an obvious changing trend from 384 summer to winter. Our effort will encourage more metagenomics applications, 
385 but researchers need to be more cautious interpreting results and selecting 386 correct inputs.

\section{Best management practice to alleviate hospital ARG issues}

388 All results clearly demonstrate the antibiotic resistances dissemination via 389 aerosol was a concern in hospital. Some groups also modeled the 390 microorganism concentrations with other related factors, such as human 391 occupancy, visiting patients among departments, indoor temperature etc. $392[8,51]$, but the results only showed weak correlations. Since our study site is 393 highly compacted and intensively used every day, strict control on visitors flow 394 may not be applicable in most public hospitals. Therefore, constraining the 395 ARGs spread within certain area seems to be important. Some practices, such 396 as establishing anteroom inside of patients' room seem to work greatly [53]. 397 Researchers also recommended providing positive air pressure to reduce 398 pathogenic transmissions [7,53] as they found humidity and outdoor air 399 fractions had strong temporal patterns and strong correlations between rooms 400 [7], which was also confirmed in this study. Besides, hospital indoor water sink

401 is another potential aerosol ARG source as discovered by Kotay et al. [72].

402 The splashed droplets could lead to the transmission from contaminated hand 403 washing sinks, which was also supported by our PCR based resistome 404 detection method. Additionally, determining cleaning strategies according to 
specific microbial resistome carriers might be important, however, unlike

406 bacterial resistome, we found current metagenomic sequencing approach

407 cannot identify the potential Eukaryotic carriers even though studies have

408 already proved some resistance like kanamycin can be carried by eukaryotic

409 cells and can be transferred between prokaryotes and eukaryotes [73,74].

410 Therefore, such lack of advanced eukaryotic carrier knowledge might be a

411 factor that hinders the application of efficient cleaning strategies.

\section{Limitations and future directions}

413 Even our culture-facilitated comparative metagenomics method seemed to

414 work well in resistome patterns, Illumina sequencing still needs more

415 turnaround time (lab work and analysis). However, in hospital related

416 applications the longer procedure might cause issues, especially for quick

417 disease identifications and evaluations. The short turnaround time and much

418 longer sequence read favors studying the structure and genomic context of

419 resistance determinants, which was not able to be addressed by Illumina

420 sequencing. Because antibiotic regions are often flanked by repetitive insertion

421 sequences and cannot be resolved by standard short read methods $[75,76]$.

422 Therefore, we recommended developing comparative metagenomics based

423 on the quick Nanopore sequencing to perform hospital applications in future.

424 Meanwhile, a yearlong temporal and spatial micro-ecology trend in a newly 
425 opened hospital suggested there was basically no stable universal bacteria

426 transmission pattern within hospital [8]. Even though our results correlated to

427 other aerosolized findings in China (Table S4), we still speculated that our one

428 year survey only demonstrated limited aerosol ARG patterns, meaning multiple

429 survey years and more sampling frequencies were needed in future in order to

430 identify a stable pattern in large-size hospitals.

\section{Conclusion}

432 The overwhelmingly present ARGs and bacterial carriers in hospital clearly

433 suggest an urgent threaten to human health. The seasonal ARG and

434 micro-ecology changes identified in our study delineated a potential plasmid

435 mediated ARG transferring among departments temporally and spatially.

436 Traditional detection methods, e.g. cultural and PCR related technologies etc.

437 justified the metagenomics approaches, and the state-of-art analyzing

438 methods with strict cutoffs are able to assisting metagenomics approaches to

439 providing more accurate interpretations.

\section{Methods}

\section{Site description, sample collection and handling}

442 Two sampling rounds were performed on summer (Aug. 2018) and winter (Jan.

443 2019) at Shenzhen Hospital of Peking University, respectively (only two 
444 distinct seasons based on ambient temperature at Shenzhen city). The

445 hospital was operated at the end of 1999 and serves over 8000 persons daily.

446 It has around 50 functional departments, and the total visitors reached 2.92

447 million in 2018. Three types of samples were surveyed in this study, including:

448 1) inlet and outlet waters from fountains in Outpatient hall/Main entrance (abbr.

449 as M), Ophthalmology (abbr. as O) and Pediatrics (abbr. as P) departments; 2)

450 door handle and lobby chair surface swabs collected using sterilized cotton

451 from $\mathrm{M}, \mathrm{O}, \mathrm{P}$, Cancer center, Male reproductive center and Inpatient

452 departments (six departments); 3) air conditioner filters from $\mathrm{M}, \mathrm{O}$, and $\mathrm{P}$

453 departments in two distinct seasons (1 denotes summer and 2 denotes winter

454 sampling) with inpatient department filter sampled only in winter. In this

455 hospital, Outpatient hall (M) is the main entrance to all facilities/departments,

456 and Pediatrics department has more patients compared to Ophthalmology

457 department, which serves around 4000 inpatients yearly. Water samples were

458 collected in sterilized container, and cotton swabs were sampled as previously

459 described [1, 2]. As the main study targets, air conditioner filters (see "filter dirt"

460 in Figure S1) were firstly detached by cleaning expert and then transported

461 back to our lab with swabs and waters on ice within 2 hours after sampling.

462 After received at lab, the dirt on filters were shaken and washed off into

463 sterilized autoclavable containers using sterilized $\mathrm{ddH} 2 \mathrm{O}$. Thereafter, the 
464 nonsoluble and dissolved dirt samples were filtrated immediately using 0.22

$465 \mu \mathrm{m}$ filters (Millipore, USA). Cotton swabs and the filtrated membranes were 466 then transferred to purple-capped tubes in FastDNA kit (MP Biomedicals, USA) 467 and stored at $-20^{\circ} \mathrm{C}$ before downstream DNA extractions (see Figure S1 for 468 overall schematic overview).

\section{Metagenomics sequencing data analyses}

470 For cultivation, DNA extraction, PCR and sequencing lab work, please refer to 471 supporting information (SI). We used Illumina HiseqXten-PE150 platform to 472 perform aerosol metagenomics sequencing (Novogene, Nanjing, China). The 473 sequencing library size was $350 \mathrm{bp}$ and $10 \mathrm{G}$ raw data was initially aimed (see 474 Table S7). Each department was designated to have two samples. We also 475 performed two replicates in $M$ and $P$ on winter sampling to testify the 476 reproducibility of our methodology, and one randomly picked replicate from M 477 and $P$ winter samples was used for subsequent analysis. In this study, 478 metagenomics analyses were based on reads and metagenome-assemblies, 479 respectively. All reads were QC qualified before application [28]. The following 480 four subsections focused on read level analyses, and the last subsection dealt 481 with assembly analyses.

482 ARG detection 
483 The rapid characterization and quantification of ARGs were analyzed by 484 ARGs-OAP v2.0, which was developed by Yin et al [29]. Briefly, both $16 \mathrm{~S}$ and 485 ARG type/subtype information were extracted from metagenomics reads at 486 first. And then, the normalized ARG profiling was calculated by normalizing the 487 extracted ARG-sequences against reads mapped to 16S rRNA gene (V6 488 region). Both ARG type and subtype (equivalent to genotype) results were 489 summarized in SI File 1.

\section{Community structure and functionality analysis}

491 MetaPhIAn2 was used to compute the composition of microbial communities 492 with species-level resolution [30]. Since MetaPhIAn2 can generate 493 species-level resolution, we searched the pathogen species in the microbial 494 community profile to generate an aerosol pathogen table with abundance 495 information (SI File 2). The community profiling results can be visualized in 496 Figure S2. We also applied HUMAnN2 to profile microbial pathways using 497 UniRef90 and ChocoPhIAn databases with the default protocols [31].

\section{8 rRNA identifications and OTU analysis}

499 In this study, we used SortMeRNA (version 2.1b) [32] to extract both $16 \mathrm{~S}$ and $50018 \mathrm{~S}$ rRNA sequences from metagenomics reads. silva-bac-16s-id90.fasta and 501 silva-euk-18s-id95.fasta databases were used with --fastx --paired_in --aligned 
502 output --log -a 8 parameter settings. Extracted $16 S$ and $18 S$ sequences were 503 then analyzed by vsearch (version 2.8.0) [33] to generate OTU tables for all 504 tested samples (identity cutoff $=0.97$ ). OTU were then analyzed by 505 alpha_diversity.py program in QIIME (1.9.1) [34] to calculate Good's coverage 506 and Shannon index values (a diversity).

\section{$507 \quad$ Bayesian source tracking}

508 In this study, we focused on three types of source tracking analyses using 509 SourceTracker [35]. Since patients, doctors and visitors all start their routines

510 at Outpatient hall, we assume it can serve as the "source" in Bayesian

511 algorithm (both cultivation and PCR results confirmed our assumption), leaving 512 other departments as "sink" in algorithm. Each vsearch generated OTU table 513 was firstly sorted based on abundances, and then commonly shared 514 information was extracted and reorganized into matrix format for all samples 515 (SI File 2). Besides community source tracking, we also took ARG genotype 516 profiling from ARGs-OAP v2.0 and pathogen detection tables into source 517 tracking analyses. $\quad$ SourceTracker $\quad \mathrm{R}$ code

518 (https://github.com/danknights/sourcetracker) was used to generate the bar 519 chart (Figure 2).

520 ARG carrier and plasmid identifications from metagenome-assemblies 
521 We assembled metagenomics sequencing reads separately (clustered by 522 departments). Since we have replicates in winter sampling, we assembled two 523 winter replicates with one summer sample together regarding M and P. All 524 assembly processes were performed using CLC Genomics Workbench 12 525 (Qiagen Bioinformatics, USA). The program was run by default setting with 526 minor changes: 1) the minimum contig length was set to 500 bp instead of 200 $527 \mathrm{bp} ; 2$ ) length fraction (mapping process) was set to 0.8 . After we obtained $\mathrm{M}, \mathrm{O}$ 528 and $\mathrm{P}$ aerosol metagenome-assemblies using de novo assembly method, 529 open read frames (ORFs) in each assembly were predicted by MetaGeneMark 530 [36]. Diamond [37] was then applied to search these ORFs against RefSeq 531 protein (July, 2017) database $(E=10-5)$. The blast format output of Diamond 532 was parsed by MEGAN5 [38] for taxonomic assignment. Then assembled 533 contigs were taxonomically classified to a Kingdom if more than $50 \%$ of the 534 genes within a contig were attributed to the same Kingdom. The same 535 threshold were used for Phylum and Class level taxonomies, while 0.4 was 536 applied for order, followed by 0.34 for family, 0.3 for genus and 0.25 for species 537 [39]. Taxonomic annotations generated from this homology-based method 538 were served to identify phylogenetic affiliation of ARG carrying bacteria. BlastP 539 [40] was used to search ORFs against SARG database with similarity cutoff of $54080 \%$ and alignment cutoff of $70 \%$ to identify ARGs from these 
541 metagenome-assemblies. A much stricter similarity cutoff of $>99 \%$ was used

542 when constructing the ARG genotype sharing network. Genus level carrier with

543 two seasonal sequencing read partition results (normalized after mapping

544 reads to contigs) were summarized in Table 1 [41]. In this study, PlasFlow.py

545 program was used to predict the existence of plasmids in all

546 metagenome-assemblies [42]. In order to increase result accuracy, we

547 changed --threshold probability parameter to 0.9 ( 0.7 by default [43]). The

548 assemblies were then subdivided into chromosomal and plasmid contigs, and

549 the plasmid contigs were used for further genotype sharing network analyses.

\section{ARG and plasmid identifications from isolates}

551 From cultivated LB agar plates, we randomly selected 11 isolates (antibiotics

552 added or free) for Nanopore sequencing. Briefly, the selected single bacterial

553 colony was firstly enriched in LB broth and after cell precipitation by centrifuge,

554 whole genome DNA extraction was performed as mentioned in previous

555 section. Each bacterial species was determined by Sanger sequencing

556 (targeting full length 16S rRNA fragment) before Nanopore sequencing. The

557 QC passed Nanopore sequencing fast5 files were basecalled with albacore

558 [44]. Next, porechop (https://github.com/rrwick/Porechop) was used to trim

559 adapters. The resulted fasta were assembled by Canu 1.8 [45]. Since we

560 performed Sanger sequencing, the estimated -genomeSize option in Canu 
561 was correctly imported for correction, trim and assemble steps. A recent 562 published plasmid database - PLSDB [46] was used to search plasmid-like 563 DNA fragments (>90\% similarity and 6000 bp in length for successfully 564 assembled contigs or $>2000$ bp for Nanopore raw reads). We then incorporate 565 the ARGs carried by plasmid-like DNA results into further analyses. Since 566 gene calling might not be reliable for Nanopore dataset, ResFinder 2.1 [47] 567 was used to identify ARGs from bacterial genomes/Nanopore reads $(90 \%$ 568 similarity and $60 \%$ alignment cutoff). The detail results were summarized in 569 Table S5 \& S6.

\section{Statistics and network analyses}

571 In order to confirm the reproducibility between replicates, Spearman's 572 correlation tests were performed using cor function in base-R (3.5). We 573 checked the community and ARG correlations, respectively on all replicates

574 (Figure S3). Meanwhile, to compare custom aerosol communities in different 575 sampling sites, we performed analysis of similarities (ANOSIM, R package 576 "vegan" [48]) using shared bacterial OTU matrix. For consistency, same matrix 577 was also used for source tracking. Procrustes analysis was performed (using 578 procrustes function in "vegan" package) to illustrating correlation between 579 temporal aerosol resistome and community profiling [49]. In brief, the 580 Procrustes transformation significance was calculated by measuring M2 
581 between principle coordination analysis (PCoA) plots (both based on 582 Bray-Curties distance) to a set of empirically distributed M2 values determined 583 from 999 computational permutations. In each permutation, the sum of 584 squared distances between matched samples (M2) was used to compute a p 585 value (compared to original distances).

586 Genome sharing network was constructed to check the dissemination pattern 587 of hospital aerosol resistome. In brief, 1) the two sampling events were 588 clustered by normalized outputs generated from MetaPhIAn2 and ARGs-OAP 589 v2.0; 2) only ARG hits with similarity greater than 0.99 were considered as 590 genotype sharing among above normalized datasets. We also incorporated 591 the potential plasmid information (computationally identified by PlasFlow.py 592 program) to clarify the possible ARG transfer among hospital departments via 593 plasmids. Meanwhile, the plasmid-like fragments identified from Nanopore 594 sequencing were also integrated into genotype sharing network to confirming 595 plasmid movements. The community correlating with ARG type results can be 596 seen in Figure S4, and genotype sharing network results (with bacterial genus 597 level information) can be seen in Figure 3. Besides ARG genotype sharing 598 network, we also applied similar protocol to network ARGs that were identified 599 by Nanopore isolate sequencing (Figure S5). The network graph was 600 illustrated using online diagram software called "draw.io". 


\section{Declarations}

\section{Acknowledgement}

603 Our research was funded by the start-up funding of Southern University of 604 Science and Technology (Y01296115). Also, we want to thank Center for 605 Computational Science and Engineering at Southern University of Science and 606 Technology (SUSTech) and core research facilities at SUSTech to provide 607 quality resources and services. We thank Dr. Chuanlun Zhang from 608 Department of Ocean Science and Engineering at SUSTech for generously 609 sharing the usage of QuantStudio qPCR.

610 Competing Interests

611 The authors declare no conflict of interest.

\section{Authors' contributions}

$613 \mathrm{XL}$ performed all lab work, data analyses and wrote the manuscript. ZW

614 performed cultural isolations, samplings and water filtrations; CD performed

615 samplings and filtration experiment. MZ, BZ, CZ, LZ, ZZ and $Y Y$ helped

616 sampling and culture related lab work. XY designed the whole study, and XL \&

617 XY planed data analysis processes. All authors edited the manuscript and 618 approved the final draft.

\section{Availability of data and materials}


620 Supporting information file has been deposited in Figshare

621 (https://figshare.com/articles/Supporting information/11437932). The Illumina 622 metagenomics sequencing raw reads were deposited into EMBL-EBI 623 (https://www.ebi.ac.uk/) under the following accession number 624 ERS4202782-ERS4202789. Cultural isolate Nanopre sequencing reads were 625 deposited under accessions from ERS4202826 to ERS4202836.

626 Ethics approval and consent to participate

627 The manuscript dose not report data collected from humans and animals.

\section{Consent for publication}

629 Not applicable.

\section{$630 \quad$ Funding}

631 Our research was funded by the start-up funding of Southern University of 632 Science and Technology (Y01296115).

\section{Reference}

634 1. Adedeji WA. THE TREASURE CALLED ANTIBIOTICS. Ann. Ibadan Postgrad. Med. 2016. 635 p. $56-7$.

636 2. Gould IM, Bal AM. New antibiotic agents in the pipeline and how they can help overcome 637 microbial resistance. Virulence. United States; 2013;4:185-91.

638 3. Ventola CL. The antibiotic resistance crisis: part 1: causes and threats. P T. United States; $6392015 ; 40: 277-83$.

640 4. Li J, Cao J, Zhu Y-G, Chen Q-L, Shen F, Wu Y, et al. Global Survey of Antibiotic Resistance 
641 Genes in Air. Environ Sci Technol. United States; 2018;52:10975-84.

642 5. Jiawen Xie, Ling Jin, Xiaosan Luo, Zhen Zhao and XL. Seasonal Disparities in Airborne

643 Bacteria and Associated Antibiotic Resistance Genes in PM2.5 between Urban and Rural

644 Sites. Environ Sci Technol Lett. 2018;5:74-9.

645 6. Lax S, Smith DP, Hampton-Marcell J, Owens SM, Handley KM, Scott NM, et al. Longitudinal 646 analysis of microbial interaction between humans and the indoor environment. Science. United 647 States; 2014;345:1048-52.

648 7. Lax S, Gilbert JA. Hospital-associated microbiota and implications for nosocomial infections.

649 Trends Mol Med. England; 2015;21:427-32.

650 8. Lax S, Sangwan N, Smith D, Larsen P, Handley KM, Richardson M, et al. Bacterial 651 colonization and succession in a newly opened hospital. Sci Transl Med. United States; $652 \quad 2017 ; 9$.

653 9. HAI Data and Statistics | HAI | CDC [Internet]. 2018. Available from:

654 https://www.cdc.gov/hai/data/index.html?CDC_AA_refVal=https\%3A\%2F\%2Fwww.cdc.gov\% 655 2Fhai\%2Fsurveillance\%2Findex.html

656 10. Klevens RM, Edwards JR, Richards CLJ, Horan TC, Gaynes RP, Pollock DA, et al.

657 Estimating health care-associated infections and deaths in U.S. hospitals, 2002. Public Health 658 Rep. United States; 2007;122:160-6.

659 11. Wiener-Well Y, Galuty M, Rudensky B, Schlesinger Y, Attias D, Yinnon AM. Nursing and 660 physician attire as possible source of nosocomial infections. Am J Infect Control. United States; $661 \quad 2011 ; 39: 555-9$.

662 12. Lopez P-J, Ron O, Parthasarathy P, Soothill J, Spitz L. Bacterial counts from hospital 663 doctors' ties are higher than those from shirts. Am J Infect Control. United States; 2009;37:7966480.

665 13. Treakle AM, Thom KA, Furuno JP, Strauss SM, Harris AD, Perencevich EN. Bacterial 666 contamination of health care workers' white coats. Am J Infect Control. United States; $667 \quad 2009 ; 37: 101-5$.

668 14. Marinella MA, Pierson C, Chenoweth C. The stethoscope. A potential source of 669 nosocomial infection? Arch Intern Med. United States; 1997;157:786-90.

670 15. Brady RRW, Verran J, Damani NN, Gibb AP. Review of mobile communication devices as 671 potential reservoirs of nosocomial pathogens. J Hosp Infect. England; 2009;71:295-300.

672 16. Ulger F, Esen S, Dilek A, Yanik K, Gunaydin M, Leblebicioglu H. Are we aware how 673 contaminated our mobile phones with nosocomial pathogens? Ann Clin Microbiol Antimicrob. 674 England; 2009;8:7.

675 17. Brady RRW, Wasson A, Stirling I, McAllister C, Damani NN. Is your phone bugged? The 
incidence of bacteria known to cause nosocomial infection on healthcare workers' mobile phones. J. Hosp. Infect. England; 2006. p. 123-5.

18. Akinyemi KO, Atapu AD, Adetona OO, Coker AO. The potential role of mobile phones in the spread of bacterial infections. J Infect Dev Ctries. Italy; 2009;3:628-32.

19. Bures S, Fishbain JT, Uyehara CF, Parker JM, Berg BW. Computer keyboards and faucet handles as reservoirs of nosocomial pathogens in the intensive care unit. Am J Infect Control. United States; 2000;28:465-71.

20. Solomon FB, Wadilo FW, Arota AA, Abraham YL. Antibiotic resistant airborne bacteria and their multidrug resistance pattern at University teaching referral Hospital in South Ethiopia. Ann Clin Microbiol Antimicrob. England; 2017;16:29. 21. Kennedy M, Ramsheh MY, Williams CML, Auty J, Haldar K, Abdulwhhab M, et al. Face mask sampling reveals antimicrobial resistance genes in exhaled aerosols from patients with chronic obstructive pulmonary disease and healthy volunteers. BMJ open Respir Res. England; 2018;5:e000321.

690 22. Choi JY, Zemke J, Philo SE, Bailey ES, Yondon M, Gray GC. Aerosol Sampling in a 691 Hospital Emergency Room Setting: A Complementary Surveillance Method for the Detection 692 of Respiratory Viruses. Front public Heal. Switzerland; 2018;6:174.

693 23. Lutz JK, Crawford J, Hoet AE, Wilkins JR 3rd, Lee J. Comparative performance of contact 694 plates, electrostatic wipes, swabs and a novel sampling device for the detection of 695 Staphylococcus aureus on environmental surfaces. J Appl Microbiol. England; 2013;115:1716968.

697 24. Hedin G, Rynback J, Lore B. New technique to take samples from environmental surfaces using flocked nylon swabs. J Hosp Infect. England; 2010;75:314-7.

699 25. Claro T, O'Reilly M, Daniels S, Humphreys H. Surface microbial contamination in hospitals: 700 A pilot study on methods of sampling and the use of proposed microbiologic standards. Am J 701 Infect Control. United States; 2015;43:1000-2.

702 26. Wang H, Sangwan N, Li H-Y, Su J-Q, Oyang W-Y, Zhang Z-J, et al. The antibiotic 703 resistome of swine manure is significantly altered by association with the Musca domestica 704 larvae gut microbiome. ISME J. England; 2017;11:100-11.

705 27. Bustin SA, Benes V, Garson JA, Hellemans J, Huggett J, Kubista M, et al. The MIQE 706 guidelines: minimum information for publication of quantitative real-time PCR experiments. 707 Clin Chem. United States; 2009;55:611-22.

708 28. Xia Y, Yang C, Zhang T. Microbial effects of part-stream low-frequency ultrasonic 709 pretreatment on sludge anaerobic digestion as revealed by high-throughput 710 sequencing-based metagenomics and metatranscriptomics. Biotechnol Biofuels. England; $711 \quad 2018 ; 11: 47$. 

SARG database and Hidden Markov Models for enhancement characterization and quantification of antibiotic resistance genes in environmental metagenomes. Bioinformatics. England; 2018;34:2263-70.

30. Truong DT, Franzosa EA, Tickle TL, Scholz M, Weingart G, Pasolli E, et al. MetaPhIAn2 for enhanced metagenomic taxonomic profiling. Nat. Methods. United States; 2015. p. 902-3. Species-level functional profiling of metagenomes and metatranscriptomes. Nat Methods. United States; 2018;15:962-8.

32. Kopylova E, Noe L, Touzet H. SortMeRNA: fast and accurate filtering of ribosomal RNAs in metatranscriptomic data. Bioinformatics. England; 2012;28:3211-7.

33. Rognes T, Flouri T, Nichols B, Quince C, Mahe F. VSEARCH: a versatile open source tool for metagenomics. PeerJ. United States; 2016;4:e2584. QIIME allows analysis of high-throughput community sequencing data. Nat. Methods. United States; 2010. p. 335-6.

35. Knights D, Kuczynski J, Charlson ES, Zaneveld J, Mozer MC, Collman RG, et al. Bayesian community-wide culture-independent microbial source tracking. Nat Methods. United States;

731 36. Zhu W, Lomsadze A, Borodovsky M. Ab initio gene identification in metagenomic sequences. Nucleic Acids Res. England; 2010;38:e132.

733 37. Buchfink $\mathrm{B}$, Xie $\mathrm{C}$, Huson $\mathrm{DH}$. Fast and sensitive protein alignment using DIAMOND. Nat 734 Methods. United States; 2015;12:59-60.

735 38. Huson DH, Auch AF, Qi J, Schuster SC. MEGAN analysis of metagenomic data. Genome 736 Res. United States; 2007;17:377-86.

737 39. Ishii S, Suzuki S, Norden-Krichmar TM, Tenney A, Chain PSG, Scholz MB, et al. A novel 738 metatranscriptomic approach to identify gene expression dynamics during extracellular 739 electron transfer. Nat Commun. England; 2013;4:1601.

740 40. Singh H, Raghava GPS. BLAST-based structural annotation of protein residues using 741 Protein Data Bank. Biol Direct. England; 2016;11:4.

742 41. Xiong W, Wang $Y$, Sun $Y$, Ma L, Zeng Q, Jiang X, et al. Antibiotic-mediated changes in the 743 fecal microbiome of broiler chickens define the incidence of antibiotic resistance genes.

744 Microbiome. England; 2018;6:34.

745 42. Krawczyk PS, Lipinski L, Dziembowski A. PlasFlow: predicting plasmid sequences in 746 metagenomic data using genome signatures. Nucleic Acids Res. England; 2018;46:e35. 

treatment plants revealed by Nanopore metagenomic sequencing. Microbiome. England; 2019;7:44.

44. Pomerantz A, Penafiel N, Arteaga A, Bustamante L, Pichardo F, Coloma LA, et al. Real-time DNA barcoding in a rainforest using nanopore sequencing: opportunities for rapid biodiversity assessments and local capacity building. Gigascience. United States; 2018;7.

45. Koren S, Walenz BP, Berlin K, Miller JR, Bergman NH, Phillippy AM. Canu: scalable and accurate long-read assembly via adaptive k-mer weighting and repeat separation. Genome Res. United States; 2017;27:722-36.

46. Galata V, Fehlmann T, Backes C, Keller A. PLSDB: a resource of complete bacterial plasmids. Nucleic Acids Res. England; 2019;47:D195-202.

47. Zankari E, Hasman H, Cosentino S, Vestergaard M, Rasmussen S, Lund O, et al. Identification of acquired antimicrobial resistance genes. J Antimicrob Chemother. England; 2012;67:2640-4.

48. Jari Oksanen, F. Guillaume Blanchet, Michael Friendly, Roeland Kindt, Pierre Legendre D, McGlinn, Peter R. Minchin, R. B. O'Hara, Gavin L. Simpson, Peter Solymos MHH, Stevens ES and HW. vegan: Community Ecology Package [Internet]. 2019. Available from:

764 https://cran.r-project.org/package=vegan

49. Forsberg KJ, Patel S, Gibson MK, Lauber CL, Knight R, Fierer N, et al. Bacterial phylogeny structures soil resistomes across habitats. Nature. England; 2014;509:612-6.

50. Tong $\mathrm{X}, \mathrm{Xu} \mathrm{H}$, Zou L, Cai M, Xu X, Zhao Z, et al. High diversity of airborne fungi in the hospital environment as revealed by meta-sequencing-based microbiome analysis. Sci Rep. England; 2017;7:39606.

770 51. Ping-Yun Huang, Zhi-Yuan Shi, Chi-Hao Chen, Walter Den1*, Hui-Mei Huang J-JT.

771 Airborne and Surface-Bound Microbial Contamination in Two Intensive Care Units of a Medical

772 Center in Central Taiwan. Aerosol Air Qual Res. 2013;13:1060-9.

773 52. Restrepo MI, Keyt H, Reyes LF. Aerosolized Antibiotics. Respir Care. United States; $774 \quad 2015 ; 60: 762-3$.

775 53. Araujo R, Cabral JP, Rodrigues AG. Air filtration systems and restrictive access conditions 776 improve indoor air quality in clinical units: Penicillium as a general indicator of hospital indoor 777 fungal levels. Am J Infect Control. United States; 2008;36:129-34.

778 54. Mirhoseini SH, Nikaeen M, Shamsizadeh Z, Khanahmad H. Hospital air: A potential route 779 for transmission of infections caused by beta-lactam-resistant bacteria. Am J Infect Control. 780 United States; 2016;44:898-904.

781 55. Gao X-L, Shao M-F, Wang Q, Wang L-T, Fang W-Y, Ouyang F, et al. Airborne microbial 
communities in the atmospheric environment of urban hospitals in China. J Hazard Mater. Netherlands; 2018;349:10-7.

56. Charalampous T, Kay GL, Richardson H, Aydin A, Baldan R, Jeanes C, et al. Nanopore metagenomics enables rapid clinical diagnosis of bacterial lower respiratory infection. Nat Biotechnol. United States; 2019;

787 57. Shanks OC, Kelty CA, Oshiro R, Haugland RA, Madi T, Brooks L, et al. Data Acceptance 788 Criteria for Standardized Human-Associated Fecal Source Identification Quantitative

789 Real-Time PCR Methods. Appl Environ Microbiol. United States; 2016;82:2773-82.

790 58. Paik S, Senty L, Das S, Noe JC, Munro CL, Kitten T. Identification of virulence 791 determinants for endocarditis in Streptococcus sanguinis by signature-tagged mutagenesis.

792 Infect Immun. United States; 2005;73:6064-74.

793 59. Doel JJ, Benjamin N, Hector MP, Rogers M, Allaker RP. Evaluation of bacterial nitrate 794 reduction in the human oral cavity. Eur J Oral Sci. England; 2005;113:14-9. 60. Ku SC, Hsueh PR, Yang PC, Luh KT. Clinical and microbiological characteristics of bacteremia caused by Acinetobacter Iwoffii. Eur J Clin Microbiol Infect Dis. Germany; 2000;19:501-5.

61. Knobler SL, O'Connor S, Lemon SM, Najafi M, editors. The Infectious Etiology of Chronic Diseases: Defining the Relationship, Enhancing the Research, and Mitigating the Effects: Workshop Summary. Washington (DC); 2004.

801 62. Noble RC, Overman SB. Pseudomonas stutzeri infection. A review of hospital isolates and a review of the literature. Diagn Microbiol Infect Dis. United States; 1994;19:51-6.

803 63. Greenwood SJ, Keith IR, Despres BM, Cawthorn RJ. Genetic characterization of the 804 lobster pathogen Aerococcus viridans var. homari by $16 \mathrm{~S}$ rRNA gene sequence and RAPD. 805 Dis Aquat Organ. Germany; 2005;63:237-46.

806 64. Simon Lax, Daniel Smith, Naseer Sangwan, Kim Handley, Peter Larsen, Miles Richardson, 807 Stephanie Taylor, Emily Landon, John Alverdy, Jeffrey Siegel, Brent Stephens, Rob Knight 808 and JAG. Colonization and Succession of Hospital-Associated Microbiota. Sci Transl Med. $8092017 ; 9$.

810 65. West PT, Probst AJ, Grigoriev I V, Thomas BC, Banfield JF. Genome-reconstruction for 811 eukaryotes from complex natural microbial communities. Genome Res. United States; $812 \quad 2018 ; 28: 569-80$.

813 66. Prussin AJ 2nd, Torres PJ, Shimashita J, Head SR, Bibby KJ, Kelley ST, et al. Seasonal 814 dynamics of DNA and RNA viral bioaerosol communities in a daycare center. Microbiome. 815 England; 2019;7:53.

816 67. Hospodsky D, Qian J, Nazaroff WW, Yamamoto N, Bibby K, Rismani-Yazdi H, et al. 
817 Human occupancy as a source of indoor airborne bacteria. PLoS One. United States;

$818 \quad 2012 ; 7:$ e34867.

819 68. Kramer A, Schwebke I, Kampf G. How long do nosocomial pathogens persist on inanimate

820 surfaces? A systematic review. BMC Infect Dis. England; 2006;6:130.

821 69. Moretti ML, Busso-Lopes AF, Tararam CA, Moraes R, Muraosa Y, Mikami Y, et al.

822 Airborne transmission of invasive fusariosis in patients with hematologic malignancies. PLoS

823 One. United States; 2018;13:e0196426.

824 70. King P, Pham LK, Waltz S, Sphar D, Yamamoto RT, Conrad D, et al. Longitudinal

825 Metagenomic Analysis of Hospital Air Identifies Clinically Relevant Microbes. PLoS One.

826 United States; 2016;11:e0160124.

827 71. Li Y, Liao H, Yao H. Prevalence of Antibiotic Resistance Genes in Air-Conditioning

828 Systems in Hospitals, Farms, and Residences. Int J Environ Res Public Health. Switzerland;

$8292019 ; 16$.

830 72. Kotay SM, Donlan RM, Ganim C, Barry K, Christensen BE, Mathers AJ. Droplet- Rather 831 than Aerosol-Mediated Dispersion Is the Primary Mechanism of Bacterial Transmission from

832 Contaminated Hand-Washing Sink Traps. Appl Environ Microbiol. United States; 2019;85.

833 73. Sieber KB, Bromley RE, Dunning Hotopp JC. Lateral gene transfer between prokaryotes 834 and eukaryotes. Exp Cell Res. United States; 2017;358:421-6.

835 74. Davies J, Davies D. Origins and evolution of antibiotic resistance. Microbiol Mol Biol Rev.

836 United States; 2010;74:417-33.

837 75. Wick RR, Schultz MB, Zobel J, Holt KE. Bandage: interactive visualization of de novo

838 genome assemblies. Bioinformatics. England; 2015;31:3350-2.

839 76. Monahan LG, DeMaere MZ, Cummins ML, Djordjevic SP, Roy Chowdhury P, Darling AE.

840 High contiguity genome sequence of a multidrug-resistant hospital isolate of Enterobacter

841 hormaechei. Gut Pathog. England; 2019. p. 3.

\section{FIGURE LEGEND}



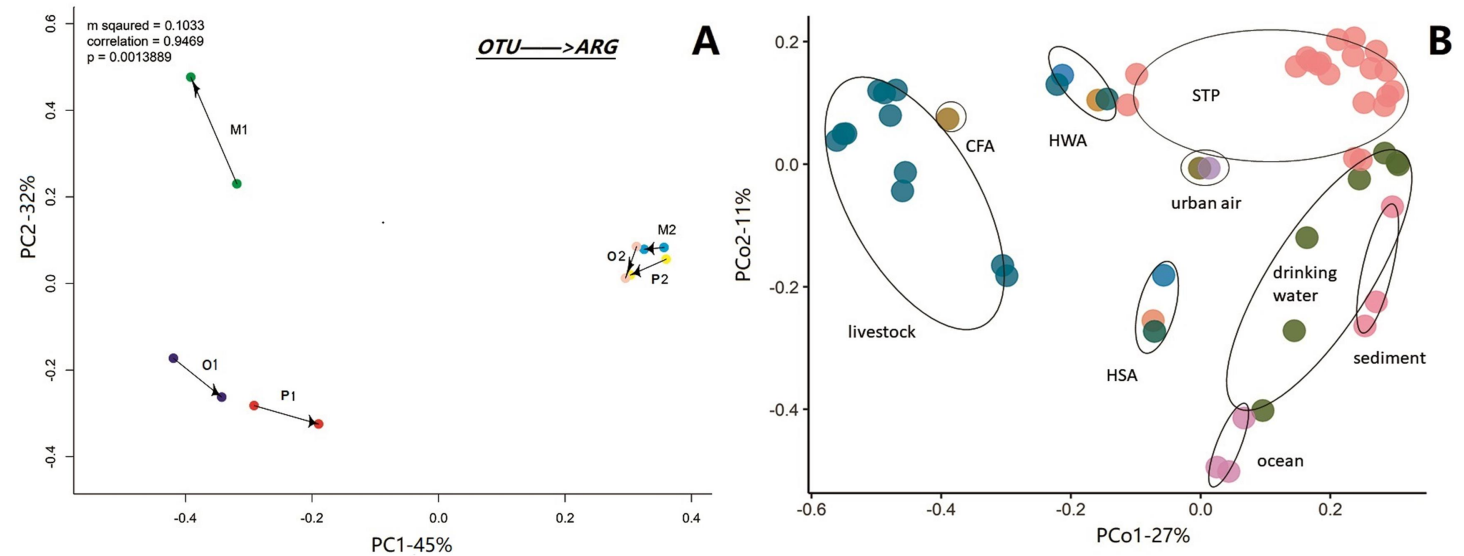

844 Figure 1 Temporal and spatial aerosolized comparative metagenomics results. Figure $1 \mathrm{~A}$ is 845 Procrustes superimposition plot that depicts confident correlation between aerosol resistome 846 (Bray-Curtis) and community composition (Bray-Curtis); Figure 1B is principal coordinate 847 analysis (PCOA) based on ARG subtypes of various environmental samples: CFA represents 848 chicken farm air, HWA is hospital winter aerosols, HSA is hospital summer aerosols; STP 849 represents sewage treatment plant.

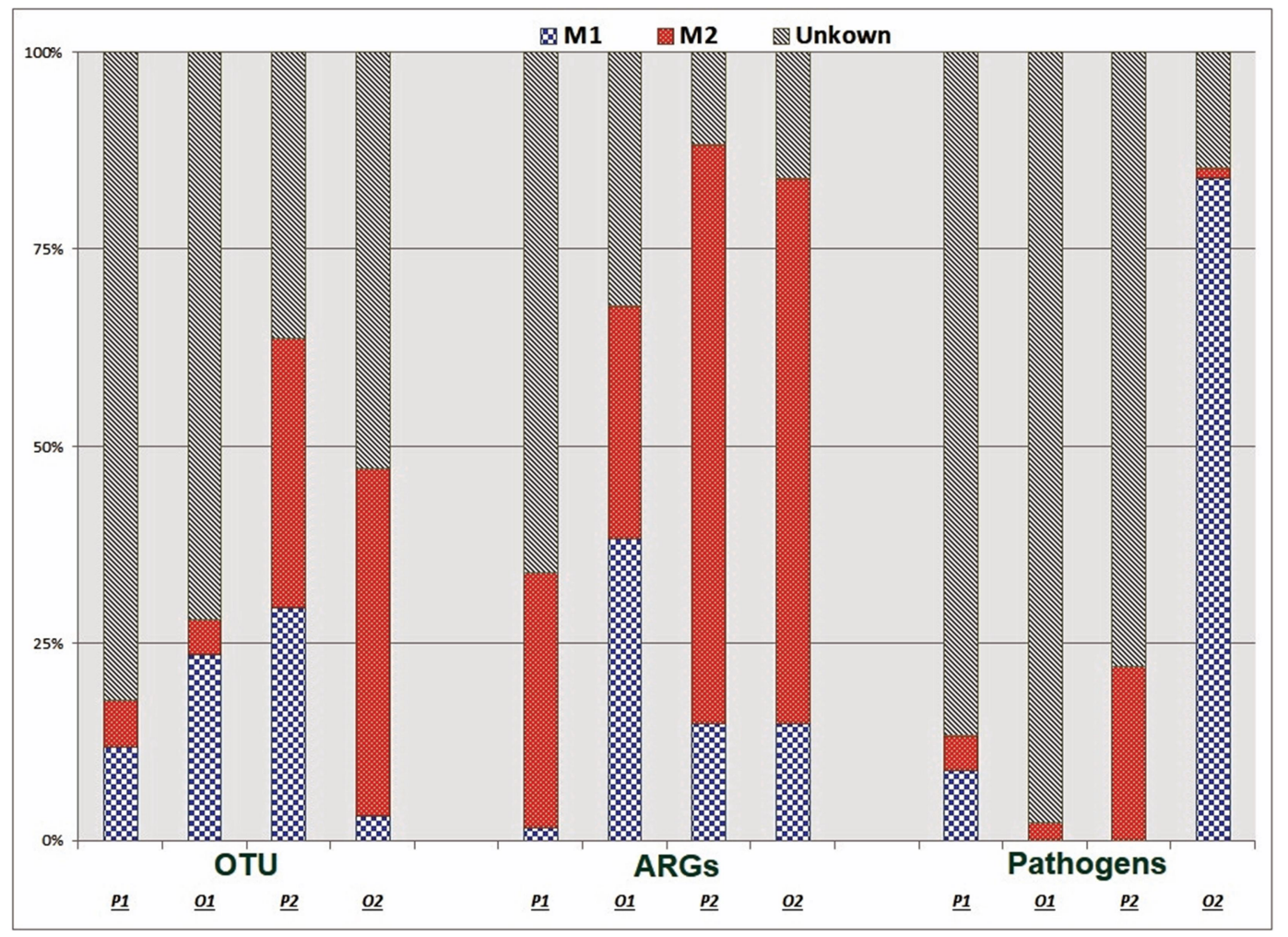

851 Figure 2 Temporal aerosol Bayesian SourceTracker algorithm results. M stands for Outpatient, 852 O represents Ophthalmology and Pediatrics is abbreviated as P. Two season's M aerosol 853 samples as source and other departments as sink for SourceTracker. 


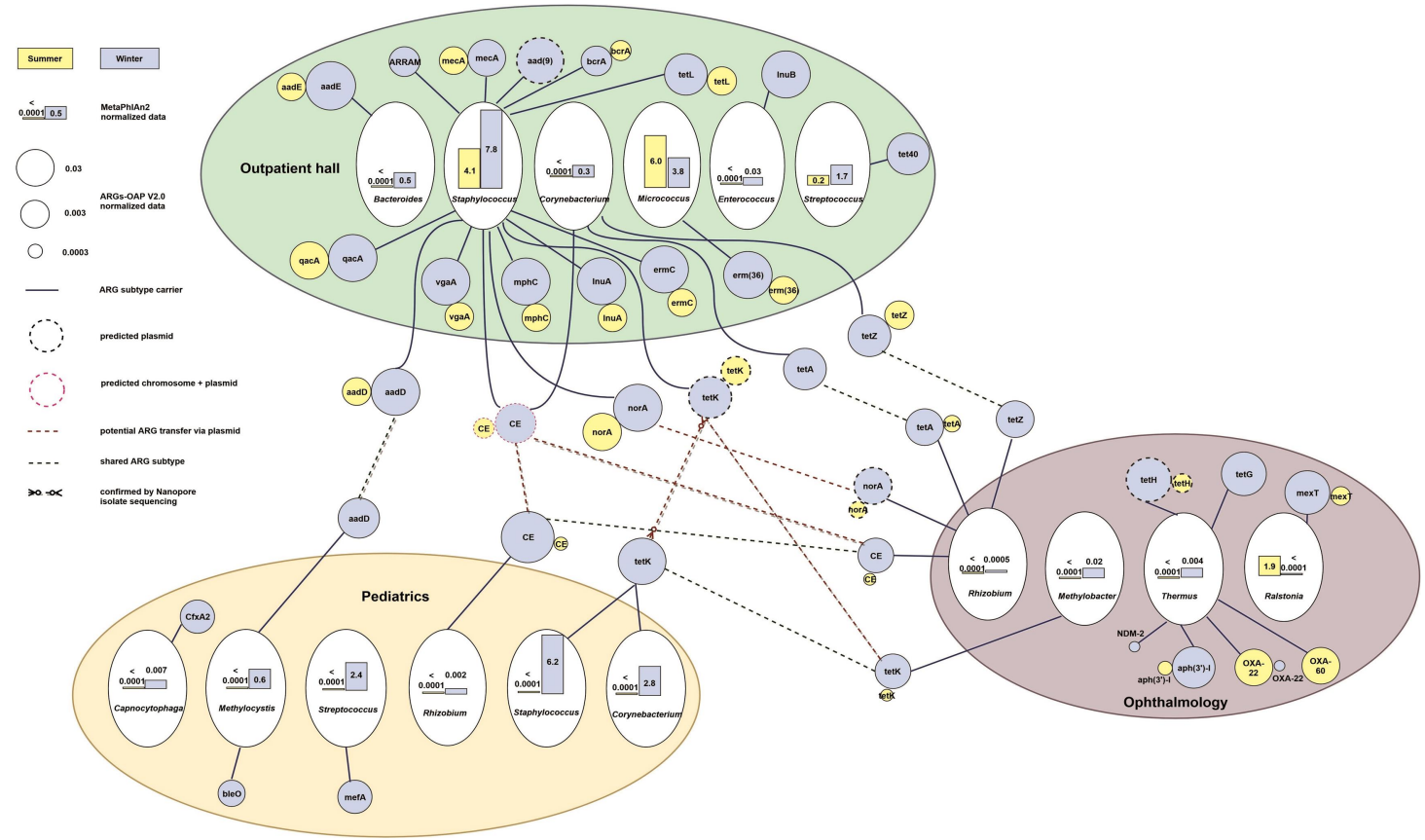

855 Figure 3 ARG genotype sharing network. Different colored oval represents different

856 departments studied. Vertical ellipse within each oval represents ARG genera that were 857 identified with their relative abundances in summer (yellow) and winter (blue), shown as bar 858 chart. Circle-pairs represent ARG genotypes. Genotypes are linked to this carrier as network 859 edges. Size of circle is proportional to its abundance (normalized against 16S rRNA gene) 860 within aerosol samples. Circle border represents the mobile mechanisms: plasmid carrying 861 (dotted line) and chromosome encoding (full line). ARG genotypes shared among departments 862 are shown in the middle area outside oval and each sharing instance was further linked with 863 dash lines; those plasmid (red) and chromosomal (green) carrying are shown as different 864 colors. Those sharing instances are confirmed by whole-genome Nanopore sequencing $865 \quad(->0----0<-)$.

\section{TABLE}

867 Table 1 Aerosol ARGs and corresponding carriers identified by metagenome-asseblies in 868 different departments. count: contig numbers; contig per: ARG-carrying contig in percentage of 869 total contig; ppm\%: the normalized part per million read percentage (see the corresponding 870 reference for more details). 


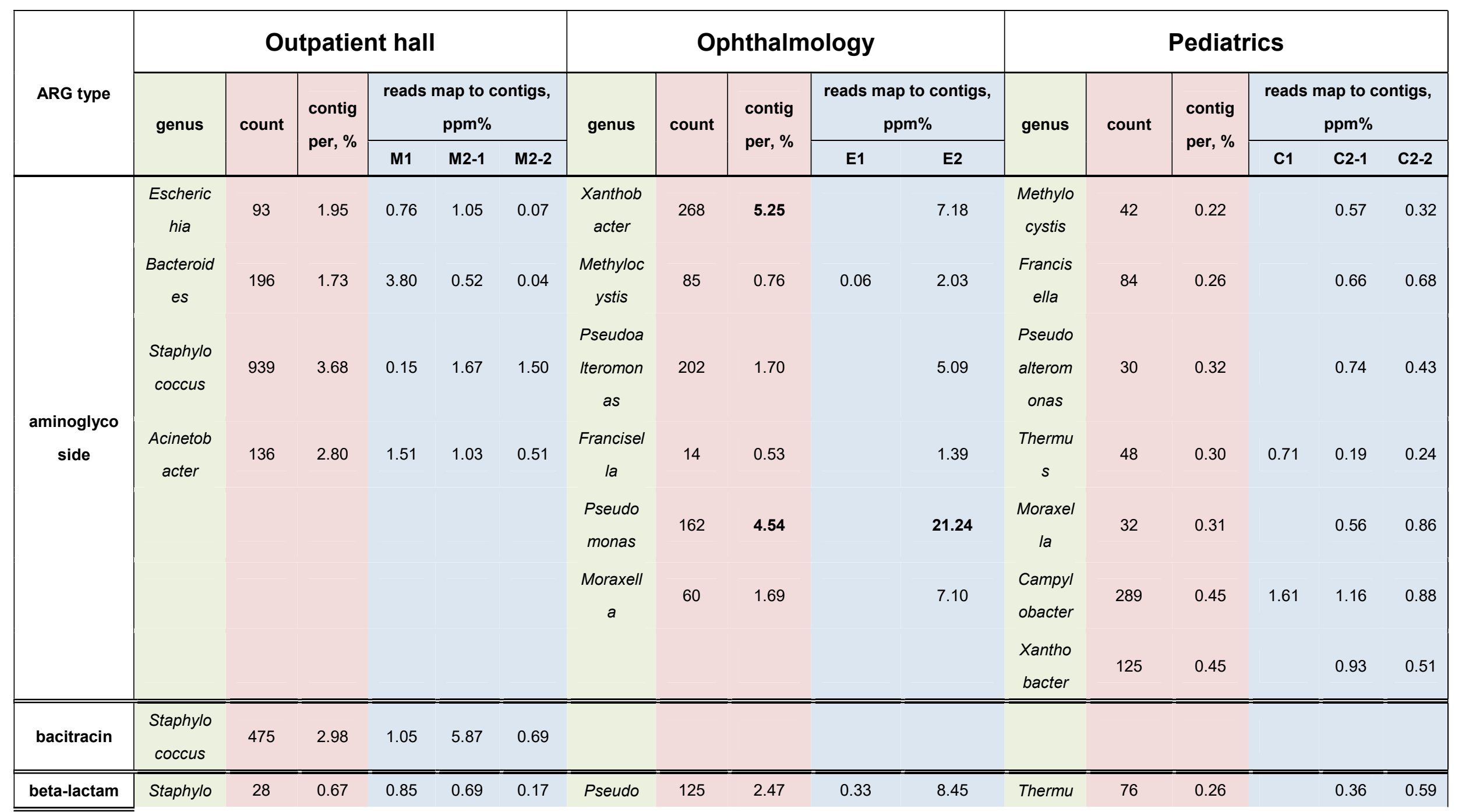




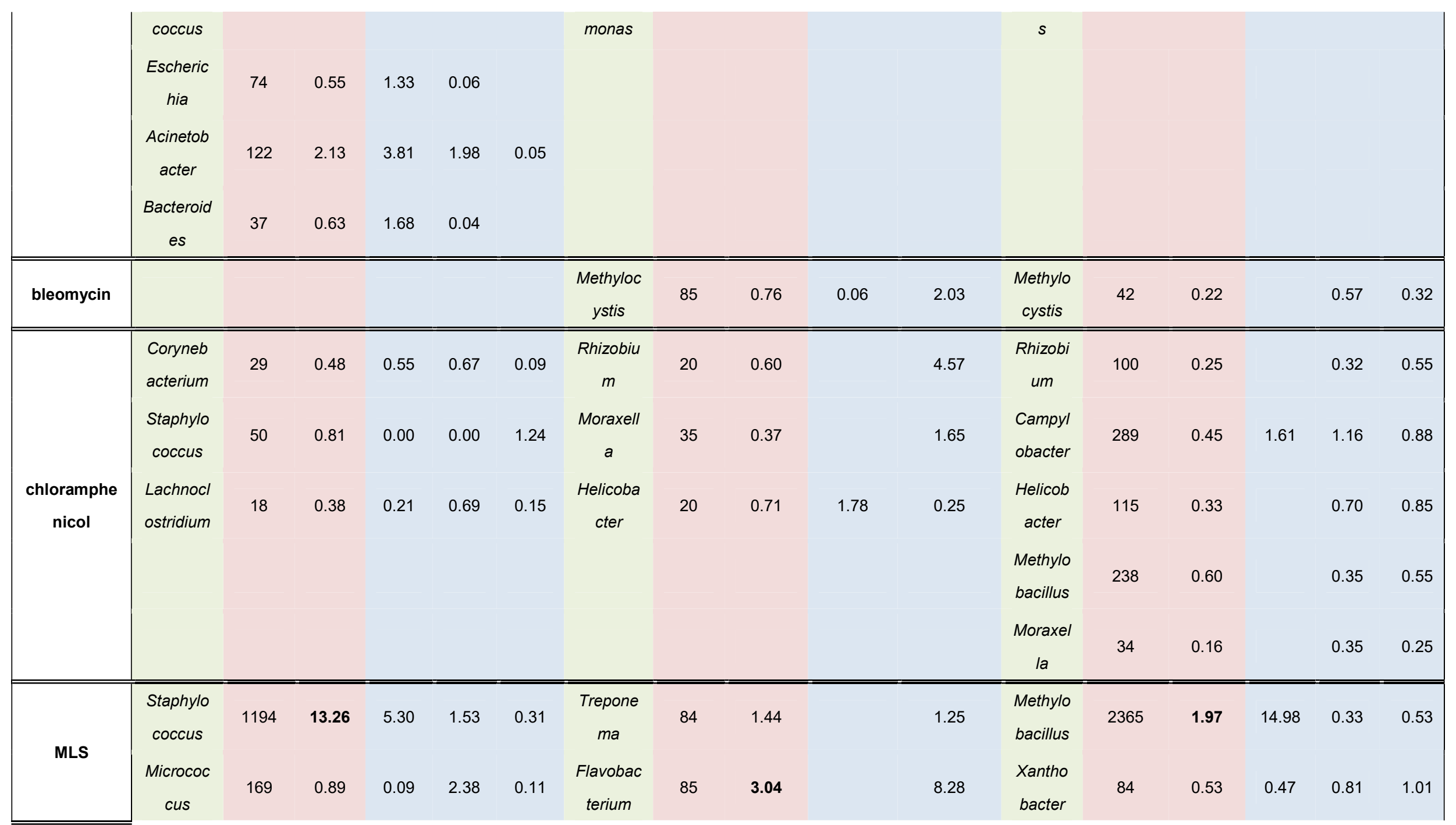




\begin{tabular}{|c|c|c|c|c|c|c|c|c|c|c|c|c|c|c|c|c|c|}
\hline & $\begin{array}{c}\text { Enteroco } \\
\text { ccus }\end{array}$ & 2558 & 11.02 & 0.00 & 0.76 & 10.21 & $\begin{array}{c}\text { Neisseri } \\
\text { a } \\
\text { Legionell } \\
\text { a }\end{array}$ & 12 & 0.37 & & 1.76 & $\begin{array}{c}\text { Neisseri } \\
\text { a } \\
\text { Trepon } \\
\text { ema }\end{array}$ & 164 & 0.98 & 10.17 & 0.13 & 0.27 \\
\hline & & & & & & & $\begin{array}{c}\text { Acidiphili } \\
\text { um } \\
\text { Xanthob } \\
\text { acter }\end{array}$ & 18 & 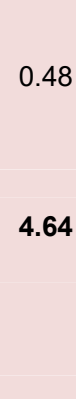 & 0.42 & 4.55 & $\begin{array}{c}\text { Pseudo } \\
\text { alterom } \\
\text { onas } \\
\text { Aeromo } \\
\text { nas } \\
\text { Paracoc } \\
\text { cus }\end{array}$ & 18 & 0.17 & 1.58 & 0.45 & 0.64 \\
\hline \multirow{4}{*}{ multidrug } & $\begin{array}{c}\text { Staphylo } \\
\text { coccus } \\
\text { Acinetob } \\
\text { acter }\end{array}$ & 466 & 7.07 & $\begin{array}{l}1.21 \\
2.30\end{array}$ & $\begin{array}{l}1.55 \\
0.16\end{array}$ & $\begin{array}{l}0.63 \\
0.02\end{array}$ & $\begin{array}{c}\text { Spirocha } \\
\text { eta } \\
\text { Alcaligen } \\
\text { es }\end{array}$ & 18 & $\begin{array}{l}0.47 \\
1.50\end{array}$ & 1.05 & 6.62 & $\begin{array}{c}\text { Ralstoni } \\
\text { a } \\
\text { Methylo } \\
\text { bacillus }\end{array}$ & 307 & $\begin{array}{l}3.01 \\
0.40\end{array}$ & 0.14 & 0.86 & 0.92 \\
\hline & & & & & & & $\begin{array}{c}\text { Legionell } \\
\text { a } \\
\text { Pseudoa } \\
\text { Iteromon } \\
\text { as }\end{array}$ & 11 & 0.32 & & 0.98 & $\begin{array}{c}\text { Rhizobi } \\
\text { um } \\
\text { Spiroch } \\
\text { aeta }\end{array}$ & 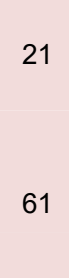 & 0.19 & 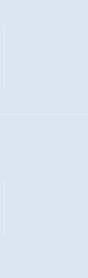 & 0.27 & 0.46 \\
\hline & & & & & & & & & & & & $\begin{array}{c}\text { Fluoriba } \\
\text { cter }\end{array}$ & 159 & 0.56 & & 0.45 & 0.52 \\
\hline & & & & & & & & & & & & Neisseri & 112 & 0.56 & 0.09 & 1.44 & 0.83 \\
\hline
\end{tabular}




\begin{tabular}{|c|c|c|c|c|c|c|c|c|c|c|c|c|c|c|c|c|}
\hline & & & & & & & & & & & & $\begin{array}{c}a \\
\text { Methylo } \\
\text { bacteriu } \\
m\end{array}$ & 117 & 0.32 & 1.29 & 1.21 \\
\hline & & & & & & & & & & & & $\begin{array}{c}\text { Alcalige } \\
\text { nes }\end{array}$ & 60 & 0.56 & 0.24 & 0.74 \\
\hline & & & & & & & & & & & & $\begin{array}{c}\text { Methylo } \\
\text { monas }\end{array}$ & 130 & 0.28 & 0.47 & 0.54 \\
\hline \multirow{2}{*}{ sulfonamide } & $\begin{array}{c}\text { Acinetob } \\
\text { acter }\end{array}$ & 157 & 1.13 & 2.56 & 0.34 & & & & & & & $\begin{array}{l}\text { Pseudo } \\
\text { monas }\end{array}$ & 66 & 0.23 & 0.46 & 0.47 \\
\hline & & & & & & & & & & & & $\begin{array}{c}\text { Blastob } \\
\text { acter }\end{array}$ & 20 & 0.24 & 0.73 & 0.36 \\
\hline \multirow{5}{*}{ tetracycline } & $\begin{array}{l}\text { Staphylo } \\
\text { coccus }\end{array}$ & 554 & 10.41 & 12.17 & 1.41 & & \multirow[t]{5}{*}{$\begin{array}{l}\text { Legionell } \\
\qquad a\end{array}$} & \multirow[t]{5}{*}{31} & \multirow[t]{3}{*}{0.94} & \multirow[t]{3}{*}{0.14} & \multirow[t]{3}{*}{15.26} & $\begin{array}{c}\text { Legione } \\
\text { Ila }\end{array}$ & 73 & 0.64 & 1.05 & 1.26 \\
\hline & $\begin{array}{c}\text { Escheric } \\
\text { hia }\end{array}$ & 28 & 0.52 & 0.20 & 2.38 & 0.18 & & & & & & $\begin{array}{c}\text { Methylo } \\
\text { bacter }\end{array}$ & 57 & 0.28 & 0.37 & 0.66 \\
\hline & $\begin{array}{c}\text { Acinetob } \\
\text { acter }\end{array}$ & 2699 & 12.19 & 23.86 & 3.83 & 0.39 & & & & & & $\begin{array}{c}\text { Rhizobi } \\
\text { um }\end{array}$ & 149 & 0.80 & 0.48 & 0.57 \\
\hline & $\begin{array}{l}\text { Coryneb } \\
\text { acterium }\end{array}$ & 147 & 1.62 & 1.76 & 0.30 & 0.08 & & & & & & $\begin{array}{c}\text { Methylo } \\
\text { monas }\end{array}$ & 42 & 0.44 & 1.03 & 0.97 \\
\hline & $\begin{array}{c}\text { Streptoco } \\
\text { ccus }\end{array}$ & 33 & 0.43 & 0.79 & 0.35 & & & & & & & $\begin{array}{l}\text { Methylo } \\
\text { bacillus }\end{array}$ & 454 & 1.83 & 3.70 & 3.05 \\
\hline trimethopri & Escheric & 61 & 1.28 & 2.17 & 1.16 & 0.07 & & & & & & Francis & 48 & 0.52 & 0.87 & 0.86 \\
\hline
\end{tabular}


m

hia

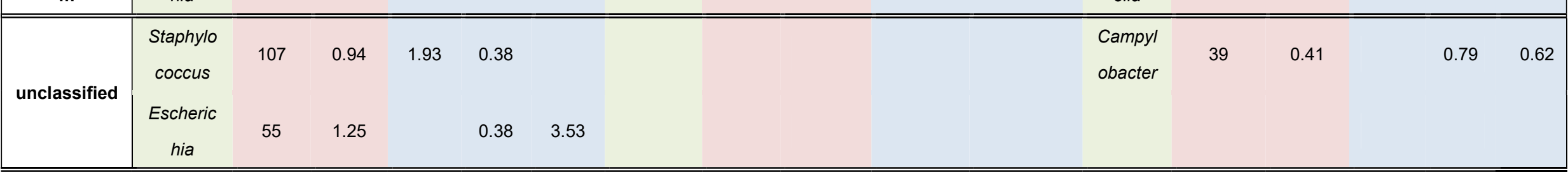




\section{Figures}
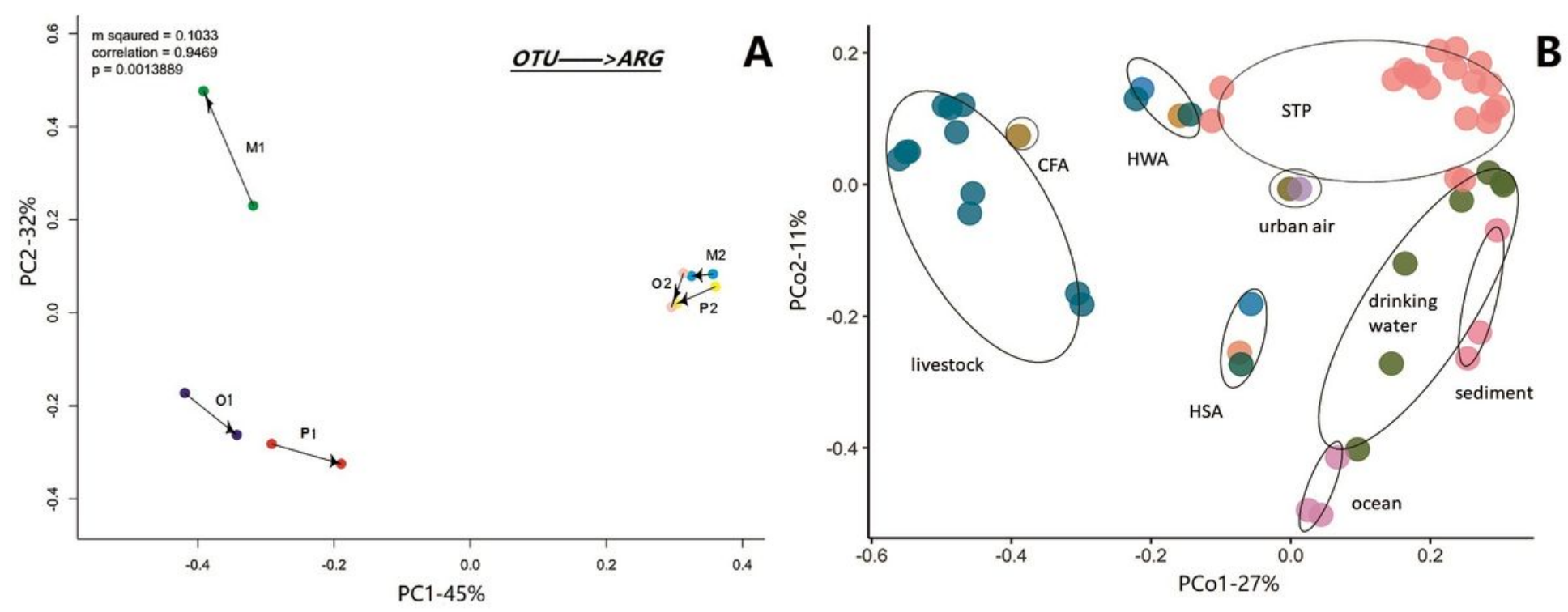

Figure 1

Temporal and spatial aerosolized comparative metagenomics results. Figure $1 \mathrm{~A}$ is Procrustes superimposition plot that depicts confident correlation between aerosol resistome (Bray-Curtis) and community composition (Bray-Curtis); Figure 1B is principal coordinate analysis (PCoA) based on ARG subtypes of various environmental samples: CFA represents chicken farm air, HWA is hospital winter aerosols, HSA is hospital summer aerosols; STP represents sewage treatment plant. 


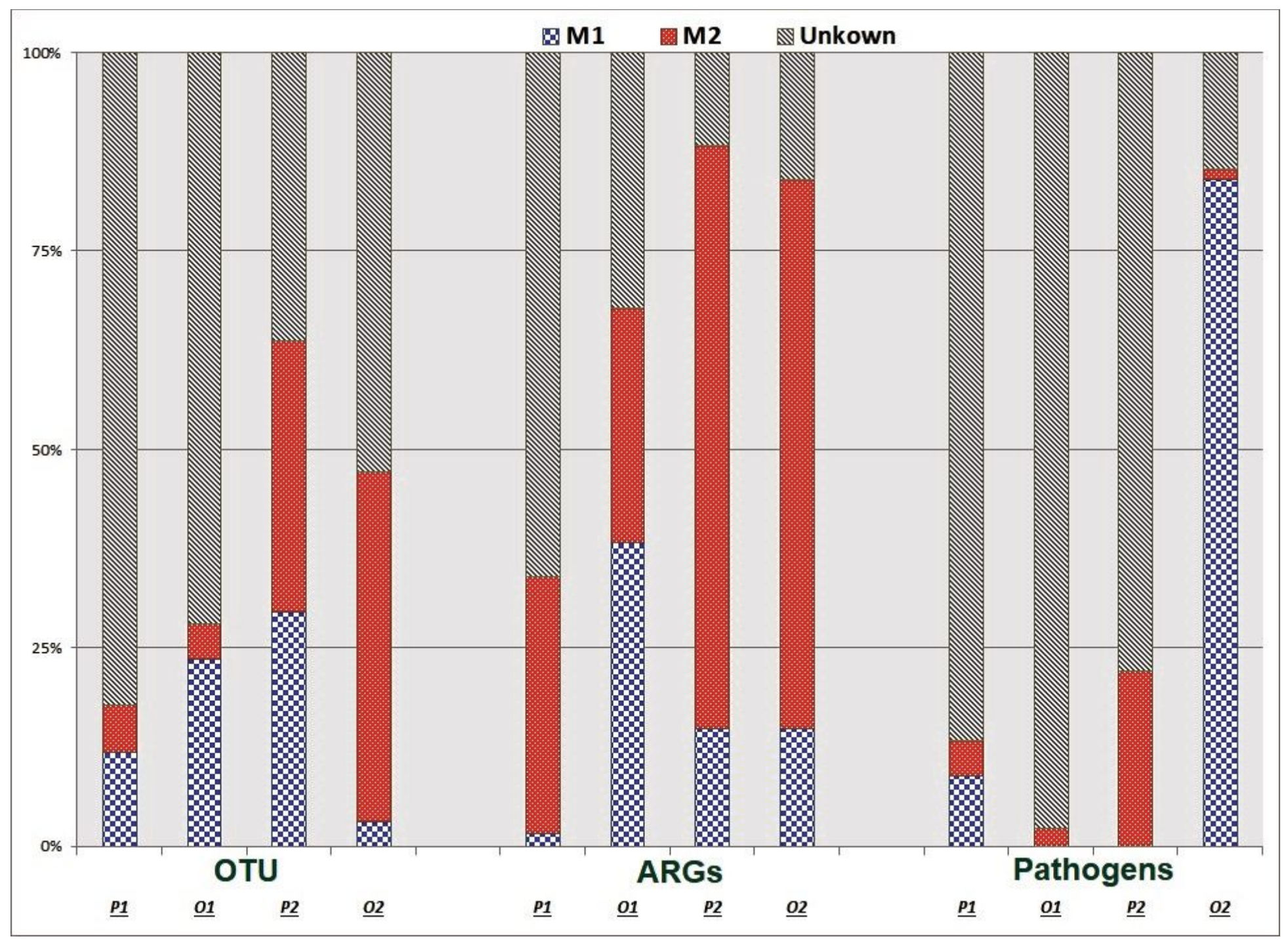

Figure 2

Temporal aerosol Bayesian SourceTracker algorithm results. $M$ stands for Outpatient, 0 represents Ophthalmology and Pediatrics is abbreviated as P. Two season's M aerosol samples as source and other departments as sink for SourceTracker. 


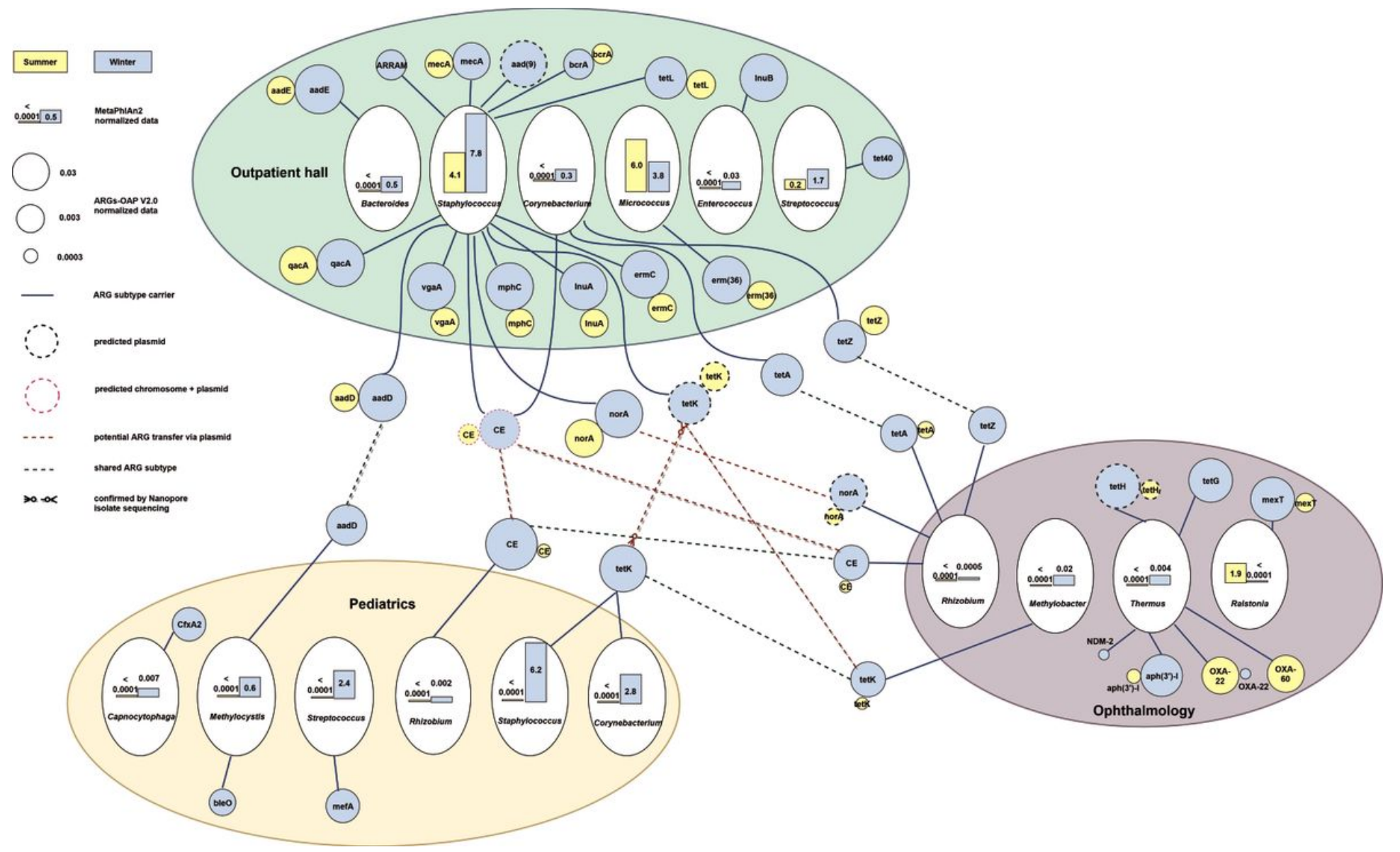

Figure 3

ARG genotype sharing network. Different colored oval represents different departments studied. Vertical ellipse within each oval represents ARG genera that were identified with their relative abundances in summer (yellow) and winter (blue), shown as bar chart. Circle-pairs represent ARG genotypes. Genotypes are linked to this carrier as network edges. Size of circle is proportional to its abundance (normalized against 16S rRNA gene) within aerosol samples. Circle border represents the mobile mechanisms: plasmid carrying (dotted line) and chromosome encoding (full line). ARG genotypes shared among departments are shown in the middle area outside oval and each sharing instance was further linked with dash lines; those plasmid (red) and chromosomal (green) carrying are shown as different colors. Those sharing instances are confirmed by whole-genome Nanopore sequencing (->0---0<-).

\section{Supplementary Files}

This is a list of supplementary files associated with this preprint. Click to download.

- SIfile3HUMAnN2results.xlsx

- Table1.xlsx

- SIfile2pathogenandOTUlist.xlsx

- SIfile116SnormalizedARGsubtype.xlsx 